\title{
MECANISMOS DE NUTRICIÓN ANIMAL PARA REDUCIR EL EFECTO INVERNADERO
}

\author{
ANIMAL NUTRITION MECHANISMS TO REDUCE THE GREENHOUSE \\ EFFECT
}

\author{
Francis Liliana Valencia Trujillo \\ Zootecnista MSc. \\ Universidad Nacional Abierta y a Distancia - ECAPMA \\ ORCID https://orcid.org/0000-0001-5382-2823 \\ francis.valencia@unad.edu.co
}

Tomás Andrés Rojas López

Zootecnista

Universidad Nacional Abierta y a Distancia - UNAD

fasj2@hotmail.com

\section{RESUMEN.}

El presente documento explica los diferentes mecanismos de nutrición animal que actualmente existen, para minimizar la producción de gases de efecto invernadero que son generados en gran medida por los rumiantes, este es un problema que aumenta de gran manera, y la nutrición animal como actividad antropogénica se convierte en una generadora de gases de efecto invernadero - GEI- considerándose a la ganadería como la principal fuente de emisiones de estos gases, principalmente de Metano ( $\mathrm{CH} 4)$, en mayor proporción que otras actividades pecuarias. Con este fin,

Se concluyó que el método más adecuado que contribuye considerablemente a la reducción del efecto invernadero es el de Inclusión de leguminosas en sistemas silvopastoriles principalmente con Leucaena leucocephala puesto que es la leguminosa que mejores ventajas nutricionales presenta por su contenido de taninos inhibe la producción de $\mathrm{CH} 4$ con reducción de porcentajes de hasta un $15 \%$ en la producción de gases de efecto invernadero por unidad de producto y una disminución del $20 \%$ por kg de Materia Seca consumida o degradada.

Palabras Claves: Efecto invernadero; nutrición animal; gases; mecanismos; alimentación; ganadería; leguminosas; leucaena.

DOI: https://doi.org/10.22490/ECAPMA.3459 


\section{ABSTRACT.}

This bibliographical review explains the different mechanisms of animal nutrition that exist to reduce the emission of greenhouse gases that are generated to a great extent by ruminants, this is a problem that increases greatly, and animal nutrition as an anthropogenic activity. It converts into a generator of greenhouse gases (GGE), considering livestock as the main source of emissions of these gases, mainly methane $(\mathrm{CH} 4)$, in greater proportion than other livestock activities.

It is concluded that the most suitable method that contributes significantly to the reduction of the greenhouse effect is the inclusion of legumes in silvopastoral systems mainly with Leucaena leucocephala since it is the legume that has the best nutritional advantages due to its content of condensed tannins (TC) inhibits the methane production with percentages of up to $15 \%$ less greenhouse gas emissions per unit of product and around 20 less per kilogram of DM consumed or degraded.

Key words: Greenhouse effect; animal nutrition; gases; mechanisms; Food; livestock; legumes; leucaena.

\section{INTRODUCCIÓN.}

En los últimos años se han llevado a cabo investigaciones tendientes a encontrar estrategias efectivas en la disminución de las producciones de gas metano a nivel pecuario. Estas estrategias de disminución se pueden clasificar dentro de tres ramas de manipulación, que no son excluyentes una de la otra, como son, la manipulación animal como tal, la manipulación nutricional y la manipulación microbiana. Es de resaltar, que cualquier método de reducción de las producciones de gas metano se realiza por medio de la alteración del ecosistema ruminal afectando así la tasa de metanogénesis. La conveniencia de las formas de manipulación de la producción de gas metano, dependerá en gran medida de la forma de producción de la ganadería.

Este trabajo recopila los distintos mecanismos que hay en la nutrición animal para mitigar las producciones de gas metano en la nutrición de ganado bovino, sus ventajas y la posibilidad de que sean implementadas en muchos sistemas productivos del país y del mundo, generando así un impacto social, económico y ambiental positivo. 


\section{La ganadería como generadora de Gases de Efecto Invernadero}

La producción de gases Efecto Invernadero (GEI) en la ganadería bovina, se presentan bajo las formas de gas metano (44\%), óxido nitroso $(29 \%)$ y dióxido de carbono (27\%). El gas metano, aporta 21 veces más al calentamiento global que el dióxido de carbono. (IPCC, 2007), este gas es producido por la fermentación anaeróbica de las bacterias arqueas presentes en el rumen del bovino. Se ha descubierto que, al suministrar dietas con altos contenidos de carbohidratos estructurales como los pastos, la producción del metano aumenta considerablemente, esto se debe a que la fibra contenido en ese material fibroso propicia la formación de acetato, que provee grupos metilo como sustratos para la formación del metano. El óxido nitroso, posee 310 veces mayor poder aportante al calentamiento global que el Dióxido de carbono (IPCC, 2007), y es producido igual por bacterias presentes en el rumen de los bovinos, al desdoblar los compuestos nitrogenados presentes en la dieta. Estos procesos se potencian al incorporar fuentes nitrogenadas al suelo, bajo la forma de fertilizantes para pasturas, o como orina y excretas durante el pastoreo del ganado. (Haro, Gómez, 2018).

\section{METODOLOGÍA}

La investigación se desarrolló como revisión sistémica, realizando una exploración completa de los estudios que se han realizado sobre el tema, en los últimos años, comparando los diferentes mecanismos de nutrición animal que existen para reducir el efecto invernadero.

Para obtener la información se utilizó la herramienta Google académico realizando una revisión bibliográfica de revistas científicas, artículos y páginas web de los últimos diez años, tomando como base para la búsqueda palabras claves que eran acordes a la investigación, efecto invernadero, nutrición animal mecanismos fueron algunas de las que se usaron para dicha labor. La búsqueda identificó trabajos donde se compararon diferentes métodos como Taninos, Flavonoides, Saponinas y aceites esenciales presentes en algunas plantas leguminosas; uso de lípidos y mejoramiento de la calidad del forraje hasta llegar a mejores alternativas en la nutrición de los bovinos. Se revisaron bases de datos bibliográficas, dando como resultado artículos calificados como científicos con calidad aceptable para el tema del estudio. 


\section{DISCUSION}

\section{Alternativas nutricionales para minimizar las producciones de Gases con Efecto Invernadero.}

\section{Taninos}

Los taninos hacen parte de los Metabolitos Secundarios presentes en las Plantas, y son sustancias complejas de compuestos poli fenólicos, dependiendo de su estructura química y peso molecular, tienen propiedades bacteriostáticas y bactericidas y alteran la fermentación ruminal mediante inactivación enzimática; formando complejos con las proteínas, aminoácidos y polisacáridos. Pueden presentar efectos benéficos o negativos dependiendo de su concentración en la dieta, la naturaleza de su origen, la especie animal y el estado fisiológico del animal (Makkar, 2003 citado en el artículo de alayón-Gamboa et al 2018).

Al mezclarse con las proteínas y polisacáridos los taninos forman complejos que disminuyen la digestibilidad de la materia seca y la elaboración de hidróxido metabólico, el cual es utilizado por las bacterias metanogénicas en la producción de dióxido de Carbono y metano. Estudios in vitro plantean que se pueden llegar a disminuir el metano en el rumen hasta un $63 \%$ agregando taninos, y en los estudios in vivo se han observado disminuciones de hasta un $58 \%$ en la producción de metano. Entonces, se concluye que al incorporar taninos en la alimentación de los bovinos en concentración de 3-6\% de MS, permite disminuir las emanaciones de gas metano, además incrementa la ganancia de peso y la producción de leche. (Piñeiro-Vásquez et al 2015).

\section{Saponinas}

Otro compuesto de los Metabolitos Secundarios de presentes en las Plantas que se ha estudiado con creciente interés, son las Saponinas. Las evidencias de sus efectos en la inhibición del gas Metano producido en el rumen son muy variadas. Se ven influenciadas por la dosis utilizada, su origen y composición química, y la dieta en la que se encuentra como complemento. La evidencia reciente sugiere que su incorporación a las dietas reduce considerablemente la producción de gas metano debido a una disminución en el número de protozoarios y/o una reducción en las arqueas metanogénicas (Bhatta, 2015 citado en el artículo alayónGamboa et al 2018). Pero existen investigaciones que afirman que a pesar de que se puede reducir en 20 \% la producción de gas metano al usar 
saponinas de Sapindus mukorossi o S. saponaria (Agarwal et al., 2006; Hess et al., 2004 citado en el artículo alayón-Gamboa et al 2018), no se reduce el número de protozoarios y de metanógenas; similares respuestas observaron (Albores-Moreno et al. 2017 citado en el artículo alayón-Gamboa et al 2018). al incorporar dosis crecientes de saponinas de Enterolobium cyclocarpum. Se sospecha que la falta de efecto en la defaunación ruminal se debe a la adaptación (destoxificación) que desarrollan los protozoarios ante una constante exposición a estos compuestos. Por otro lado, la actividad de inhibición que ejercen las saponinas sobre la metanogénesis se relaciona directamente con el tipo de dieta que se les suministre a los animales. Así se ha encontrado que las saponinas de Sesbania sesban ejercen un efecto de inhibición en la elaboración de gas metano, por una disminución en la población de bacterias arqueas (78 \%). Este efecto es más pronunciado cuando se adicionan a dietas a base de concentrados que con dietas a base de forrajes (Goel et al., 2008 citado en el artículo alayón-Gamboa et al 2018).

\section{Aceites Esenciales (AE)}

La utilidad principal de los aceites esenciales es proveerle a la planta defensa contra causantes de estrés, tanto abióticos como bióticos, pero también son potentes atrayentes de animales polinizadores. (Wink M, Schimmer O. citado en el artículo de Polin, Muro, Díaz 2014).

Algunas investigaciones han concluído que la utilización de los Aceites Esenciales en la dieta de bovinos reduce la producción de metano, pero además se evidencia la disminución de ácidos grasos o volátiles (AGV) totales. Ejemplos de aceites esenciales usados con este objetivo son aceites de, canela, timol ajo, orégano, rábano, entre muchos otros; todos estos han probado disminuir la generación de metano in vitro. Se presentaron casos donde la suspensión de metano se logró a través de dosis elevadas (>300 mg/L de cultivo ruminal), y en otros casos se redujo por la poca producción de ácidos grasos o volátiles o diferencia en sus proporciones.(Polin et al 2014).

\section{Inclusión de leguminosas en sistemas silvopastoriles}

(Rivera et al 2016) citado en el artículo de (Rivera, Molina et al 2017), informaron según su investigación, que en sistemas silvopastoriles de Leucaena se reduce la intensidad de las producciones de gas metano gracias a su alta calidad de la dieta y a la baja dependencia de insumos externos (como fertilizantes). Asimismo, (Naranjo et al 2012 citado en el artículo de Rivera, Molina et al 2017) estimaron que, en condiciones de alta número de árboles en la zona de pastoreo, el balance entre emisiones 
y remociones puede llegar a ser negativo, gracias a que en los sistemas silvopastoriles se puede capturar entre 17 y 32 t de CO2 ha-1 año-1 y producir cerca de 12 t de CO2 ha por año.

\section{Carbohidratos no fibrosos o fibra presente en la dieta.}

Los ácidos grasos volátiles que se generan en la cavidad ruminal no se estiman como fuente principal para la metanogénesis, ya que la transformación de $\mathrm{CO} 2$ e hidrógeno es un procedimiento que lleva tiempo (Hobson y Stewart, 1997 citado en el artículo de Aguiar, rojas 2014) por lo anterior, el gas metano localizado en la cavidad ruminal se genera por la liberación de $\mathrm{CO} 2$ e hidrógeno, estos se producen al generarse los ácidos grasos volátiles mediante la fermentación de los carbohidratos (Hungate, Smith, Bauchop, Yu y Rabinowitz, 1970 citado en el artículo de Aguiar, rojas 2014 ). La remoción del hidrógeno al formarse el gas metano es esencial para el correcto funcionamiento ruminal, puesto que el hidrógeno puede obstruir el metabolismo microbial (Sharp, Ziemer, Stern, Stahl, 1998 citado en el artículo de Aguiar, rojas 2014). Se evidencia un aumento en el metano con la digestión de la fibra, esto se debe al aumento en la cantidad de ácido acético en relación al ácido propiónico (Jhonson y Jhonson, 1995 citado en el artículo de Aguiar, rojas 2014), por lo anterior, alimentos más fibrosos como la cascarilla de soya producen mayor cantidad de gas metano.

\section{Uso de lípidos}

Una opción que ha sido muy estudiada para reducir la emisión de gas metano, es el uso de aditivos como las grasas en la dietas de los bovinos, puesto que se reducen por acción física las bacterias metanogénicas, también los lípidos ricos en ácidos grasos insaturados, son capaces de atrapar hidrógenos por medio de la biohidrogenación de estos, según (Johnson y Jhonson 1995 citado en el artículo de Aguiar, rojas 2014), lo que disminuye la disponibilidad de $\mathrm{H} 2$ para que se forme metano. Según (Hegarty, 1999 citado en el artículo de Aguiar, rojas 2014) algunas bacterias metanogénicas se encuentran estrechamente relacionadas con protozoarios, lo que constituye la estrecha relación del conteo de protozoarios y la reducción del gas metano. Esta asociación metanógenoprotozoo lo asegura (Hegarty 1999 citado en el artículo de Aguiar, rojas 2014) al aseverar que de la metanogénesis ruminal, el $37 \%$ se da por esta asociación. 


\section{Optimizar la calidad del forraje}

Los forrajes son plantas utilizadas en la alimentación animal, comúnmente en los sistemas de producción bovinos estos forrajes son de baja calidad (pajas, residuos de cosecha, o el pienso seco), lo cual conlleva a que se deba optimizar esa calidad del forraje mediante el procesamiento, mejorarando la digestibilidad efectiva de la dieta y la productividad de los animales. En los sistemas que se usan pajas gruesas de millo, sorgo y maíz hay una calidad nutricional mucho mayor que las pajas delgadas como las de la cebada). Y el trigo. Acciones como el manejo del pastoreo y el mejorar la calidad de la alimentación optimizando las especies forrajeras puede ayudar de igual manera a la formulación de una dieta que sea más acorde en explotación extensiva, o que puede aumentar la eficiencia alimenticia y por ende la producción. Se tienen en cuenta posibles disminuciones en la magnitud de la producción de metano del 30 $\%$ en sistemas que hoy en día presentan una alimentación de muy baja calidad, también se tienen en cuenta las alternativas de reducción de metano asociadas con el manejo del pastoreo. No obstante, hay que tener en cuenta las emanaciones indirectas fuera de la explotación originadas por la producción de alimentos adicionales. (Rivera, 2016).

\section{Mejorar la dieta y sustitutos}

La dieta se puede mejorar a través de alimentos sustitutos que modifican e influencian la fermentación ruminal y por ende actúan sobre la generación de gas metano. Al suplementar la dieta con ensilaje de maíz o ensilaje de leguminosas, se disminuye la producción de metano en comparación con los ensilados de gramíneas. Los ensilajes de maíz o ensilado de maíz/leguminosa, incrementan el consumo de alimento y la producción de ganado de leche comparándolo con el ensilado de gramínea. Pero se debe tener en cuenta toda la cadena de suministro, considerando los cambios en el uso del suelo, las emisiones provenientes de la producción de cultivos, resistencia a la variabilidad climática y del mercado, la utilización de fertilizantes y los efectos netos sobre la seguridad alimentaria regional, mediante el uso del suelo y los alimentos. (Andeweg, Reisinger, 2016)

\section{Mejorar la digestibilidad y calidad del alimento}

Cuando los alimentos son de baja calidad y por ende poseen poca digestibilidad, generalmente producen altas emisiones de gas metano. El aumento de la digestibilidad y la energía de un alimento, se logra 
mediante un adecuado manejo de los forrajes, pastos mejorados y el uso de suplementos alimenticios para conseguir una dieta equilibrada. (Andeweg, Reisinger, 2016). De esta manera se pueden mejorar la absorción de nutrientes, se incrementando la productividad de los animales, su fertilidad, y disminuyendo las emisiones de gases de efecto invernadero. (Andeweg, Reisinger, 2016).

\section{CONCLUSIONES.}

Se concluye que la nutrición animal es una actividad que incide en el efecto invernadero a través de procesos determinantes como la fermentación ruminal proceso natural que se da en el organismo de los rumiantes a través de las arqueas generando metano y dióxido de carbono que luego se expulsan a través del eructo y la exhalación del bovino, el óxido nitroso es generado principalmente en la orina y excretas de los rumiantes y las dietas con carbohidratos estructurales en abundancia también generan metano. De ahí la importancia de utilizar alternativas de nutrición animal para reducir los GEI generando principalmente en el rumen procesos fermentativos sin carbono e Hidrógeno (H2) a través de una alimentación adecuada.

Los forrajes y otros piensos que se dan al ganado participan también activamente de las emisiones de gases de efecto invernadero, ya que las dietas que son suministradas a estos animales son muchas veces de baja calidad y digestibilidad o con ingredientes que tiene alto contenido de fibra, resultando esto en altas emisiones entéricas, principalmente en los sistemas de baja productividad, rumen con alta cantidad de hidrógeno y menos producción de AGV (Acidos grasos volátiles) algunos alimentos como paja, pienso seco o residuos de cosecha no son los más óptimos en cuanto a calidad se refiere, generando esto en un mala eficiencia alimenticia. Los procesos naturales que se dan en el rumen del bovino como la fermentación ruminal y entérica dependen de la alimentación suministrada por ende forrajes, concentrados y otros alimentos de poca calidad se consideran como factores de riesgo para generar gases de efecto invernadero.

Las materias primas que son empleadas en la alimentación animal, deben cumplir con altas condiciones de calidad ya que la problemática de emanaciones de gases de efecto invernadero desde la parte nutricional, se genera porque no se suministran alimentos adecuados a los animales y en el caso específico de los bovinos, las malas prácticas de nutrición en los sistemas productivos. Se debe procurar una efectiva digestibilidad de la dieta y una excelente eficiencia alimenticia mediante materias primas 
idóneas para tales procesos a través de pastos mejorados, buen uso de pastizales, suplementos alimenticios y cambio de forrajes de esta manera se genera una dieta equilibrada mejorando la absorción de nutrientes, la productividad en los animales y lo que es más importante reducción de las emanaciones totales de los gases de efecto invernadero (GEI).

\section{BIBLIOGRAFÍA.}

Agarwal N., Kamra D.N., Chaudhary L.C., Patra A.K. (2006). Effect ofSapindus mukorossi extracts on in vitro methanogenesis and fermentation characteristics in buffalo rumen liquor. Journal of Applied Animanimal Research 30: 1-4.

Aguiar, Z. E., \& Rojas, B. A. (2014). MÉTODOS UTILIZADOS PARA REDUCIR LA PRODUCCIÓN DE METANO ENDÓGENO EN RUMIANTES. Nutrición Animal Tropical, 8(2): 72-90.

Alayón-Gamboa J.A., Ayala-Burgos A.J., Solorio- Sánchez F.J., AguilarPérez C.F., Olivera-Castillo L., Ku-Vera J.C. (2017). Effects of feeding ground pods of Enterolobium cyclocarpum Jacq. Griseb on dry matter intake, rumen fermentation, and enteric methane production by Pelibuey sheep fed tropical grass. Tropical Animal Health and Production 49: 857866.

Andeweg K. \& Reisinger A., 2016. Reducing greenhouse gas emissions from livestock: Best practice and emerging options. Global Research Alliance on Agricultural Greenhouse Gases (GRA), Sustainable Agriculture Initiative (SAI)

Goel G., Makkar H.P.S., Becker K. (2008). Changes in microbial community structure, methanogenesis and rumen fermentation in response to saponin-rich fractions from different plant materials. Journalof Applied Microbiology 105: 770777

Haslam, E. (1986) Hydroxybenzoic acid and the enigma of gallic acid. In: E. E. Conn, ed. The Shikimic acid Pathway, recent advances in Phytochemistry. New York: Plenum Press. vol. 20. p. 163-200

Haro., R, J. Gómez., B, C. (2018). fontagro.org. Obtenido de fontagro.org: www.fontagro.org/wpcontent/uploads/2017/04/documento-mitigacionalta.pdf 
Hegarty, R. (1999). Reducing rumen methane emissions through elimination of rumen protozoa. Australian Journal of Agricultural Research $50,1321-1328$

Hobson, P., Stewart, C. (1997). The Rumen Microbial Ecosystem, Chapman and Hall, London, UK

Hungate, R. E., Smith, W., Bauchop T. Yu, I., Rabinowitz, J. C. (1970). Formate as an intermediate in the bovine rumen fermentation. Journal of Bacteriology. 102 (2), 389-397

IPCC, 2007: climate change 2007: impacts, adaptation and vulnerability. Contribution of working group II to the fourth assessment report of the intergovernmental panel on climate change. Cambridge Uni-versity Press, Cambridge, UK.

Johnson KA, Johnson DE. Methane emissions from cattle. J Anim Sci, (1995); 73: 2483-2492

Naranjo, J. F.; Cuartas, C. A.; Murgueitio, E.; Chará, J. \& Barahona-

Rosales, R. (2012) Balance de gases de efecto invernadero en sistemas silvopastoriles intensivos con Leucaena leucocephala en Colombia. LRRD. 24 (8). http://www.Irrd.org/ Irrd24/8/nara24150.htm

Piñeiro-Vázquez, A. T., Canul-Solís, J. R., Alayón-Gamboa, J. A., ChayCanul, A. J., Ayala-Burgos, A. J., Aguilar-Pérez, C. F., ... \& Ku-Vera, J. C. (2015). Potential of condensed tannins for the reduction of emissions of enteric methane and their effect on ruminant productivity. Archivos de Medicina Veterinaria, 47(3), 263-272.

Polin-Raygoza, L. A. Muro, R.A, Díaz-García, L-H (2014). Aceites Esenciales Modificadores de Perfiles de Fermentación Ruminal y Mitigación de Metano en Rumiantes. Rev. mex. de cienc. pecuarias vol.5 no.1, 2547.

Rivera, J.; Molina, I. C.; Donney`s, G.; Villegas, G.; Chará, J. \& BarahonaRosales, R. (2015)

Rivera, H.J.E; Molina, B.I; Chará, O.J; Murgueito, R.E; Barahona, R.R. (2017) Sistemas silvopastoriles intensivos con Leucaena leucocephala (Lam.) de Wit: alternativa productiva en el trópico ante el cambio climático. Revista Pastos y Forrajes Vol. 40, No. 3, 171-183 
Sharp, R., Ziemer, C.J., Stern, M.D., Stahl, D.A. (1998). Taxon -specific associations between protozoal and methanogens populations in the rumen and a model rumen system. FEMS Microbiology Ecology 26 (1), 71-78.

Wink M, Schimmer O. (1999) Modes of action of defensive secondary metabolites. In: Wink M editor. Functions of plant secondary metabolites and their exploitation in biotechnology. Sheffield UK: Sheffield Academic Press; $17-112$. 